\title{
MATERIALIDADES (RE)ENCONTRADAS. LA COLECCIÓN ALAMITO Y LOS COMIENZOS DE LA ANTROPOLOGÍA ROSARINA
}

\author{
Fausto Battaggia ${ }^{1}$
}

Fecha de defensa: 15 de marzo de 2019

Directora: Dra. Soledad Biasatti

Co-Director: Dr. Juan Bautista Leoni

Jurados: Lic. Diana Tamburini, Dra. Irene Dosztal

Palabras clave: museo, profesionalización, antropología rosarina, Fondo documental Alamito

\section{Presentación y fundamentación de la investigación}

En esta tesina, me propuse indagar en la conformación del Museo del Instituto de Antropología de la Universidad Nacional del Litoral (UNL) y su aporte a la profesionalización de la disciplina en Rosario. Mi interés se centró en las expediciones arqueológicas a los sitios de "El Alamito" (Provincia de Catamarca), organizadas en los años 1957 y 1958. La investigación se basó en el abordaje socio-antropológico de un cuerpo documental vinculado con esas expediciones hallado en el Museo, al que definí como "Fondo documental El Alamito". Realicé una articulación con entrevistas y un relevamiento bibliográfico general conjugando, como dice Garbulsky, un antropoanálisis (Garbulsky, 2000). Para esto, me propuse una serie de objetivos específicos de trabajo entre los que se encontraban caracterizar el período histórico y los mecanismos que intervinieron en la conformación del actual Museo de la Escuela de Antropología, y dar cuenta de las especificidades del proceso de profesionalización de la antropología a nivel local en la ciudad de Rosario.

Abordé la investigación desde una particular definición teórico-epistemológica con el uso de la primera persona del singular. En este sentido, el desafío se basó en construir conocimiento incorporando mi propia subjetividad, asumiendo que todos los sujetos somos portadores de una historia con sentido (Bianchi y Silvano, 2001). Trayectoria particular incluida, a su vez, en contextos socio-histórico-políticos que se construyen, heredan y transmiten generacionalmente.

Las primeras preguntas de lo que luego definiría como problemática comenzaron a rondar por mi cabeza en el año 2009, con el cursado de Arqueología Americana y Argentina y Metodología de la investigación II. Desde aquel entonces me pregunté por la conformación de la carrera en Rosario, sin poder escaparme de uno de los fetiches disciplinares: "la pregunta por el origen de las cosas". Sin embargo, nunca me sentí atraído por esta temática en términos meramente "académicos", sino más bien en clave de apropiación del espacio por donde transitaba. Es decir, analizar en clave histórica la Escuela de Antropología, como medio para intentar comprender y aportar a la modificación de aspectos actuales vinculados con su funcionamiento, los contenidos y las perspectivas teórico-metodológicas hegemónicas, así como el perfil profesional delineado. Esa pregunta constante me llevó a ponerme en contacto en el año 2014 con la Profesora Nélida de Grandis, en ese entonces encargada del Museo, quien amablemente me permitió sumarme al espacio y desde allí conocer con mayor profundidad la riqueza de sus historias y colecciones.

\section{Consideraciones teórico-metodológicas: acerca de los modos de abordar el pasado}

Durante la elaboración de la problemática de investigación tenía en claro que quería articular mi experiencia en el Museo con la realización de la tesina, y para esto comencé con seleccionar El Alamito. El recorrido que al interior del museo me permitió conocer que allí no solo se alojaban piezas y fichas, sino también otro conjunto de objetos no pertenecientes a la colección, pero que sin embargo estaban estrechamente vinculados con El Alamito. En este sentido, los objetos requieren de una transformación en papel (Pegoraro y Spoliansky, 2013; Podgorny, 2011; Pupio, 2013).

En el caso de la profesionalización de la antropología en Rosario, aprendí sobre las expediciones realizadas desde el Instituto que habrían desempeñado un papel central en este proceso. De esta forma, metodológicamente hablando, restringirme a investigar a partir de la "Colección El Alamito" me habría llevado a un recorrido desde donde se haría más difícil la formulación de posibles respuestas para los interrogantes en las que basé mi investigación.

Construí la categoría "Fondo documental El Alamito" como herramienta teórico-metodológica para dar cuenta de las expediciones, articulando los diferentes elementos que lo conforman (las piezas arqueológicas y sus correspondientes fichas

1 Departamento de Arqueología, Escuela de Antropología, Facultad de Humanidades y Artes, UNR. Museo Histórico Provincial "Dr. Julio Marc", Ministerio de Cultura, Provincia de Santa Fe. Dirección postal: Mitre 1466, Rosario - f battaggia@hotmail.com. 
de ingreso al museo, informes y cartas, artículos periodísticos de la época, cuadernos de campo, dibujos de piezas, planimetría de los sitios y fotografías). Esta perspectiva me permitió elaborar una serie de núcleos antropológicos de sentido desde los que dar cuenta de la profesionalización disciplinar en Rosario, como caracterizar aspectos como el marco teórico desde el que se estructuraban las investigaciones arqueológicas, las preguntas y los problemas que estructuraban los trabajos, la metodología de campo adoptada, los modos en que se conformaban los equipos y las formas de financiamiento con las que se contaba. Además, el empleo de la noción de "arqueología de museo" me permitió re-contextualizar elementos arqueológicos, re-construir sus historias, otorgando nuevos sentidos a piezas y los documentos de archivo que en apariencia parecían estar completamente desconectados.

\section{La profesionalización de la antropología en Rosario}

Alberto Rex González eligió el título metafórico de "Tiestos dispersos" para su libro de relatos autobiográficos novelados. Investigar en arqueología, sin dudas, tiene mucho de eso. Buscar, recolectar, ordenar y reordenar, interpretar y reinterpretar fragmentos desperdigados. En una entrevista realizada el 24 de octubre de 2017, la profesora María Teresa Carrara expresó: "Estás recogiendo los restos de recuerdos nuestros".

Algunos de los elementos del Fondo se hallaron en el mobiliario del Box del Departamento de Arqueología de la Escuela de Antropología, mientras que los documentos restantes estaban en el Museo de la Escuela. En el caso del mueble, sus características permitieron recuperar una suerte de columna estratigráfica conformada por una serie de documentos -niveles estratigráficos- que fueron depositados a lo largo del tiempo, debido a que los de la parte superior eran más recientes que los de la parte inferior.

Entre finales de la década de 1950 y comienzos de 1960, en Rosario se configuró un campo intelectual caracterizado por trabajos interdisciplinarios, cargados de discusiones y autocrítica (Achilli, 2014, 2015). En esta etapa coexistieron diversos sectores en pugna "conservadores antimodernizantes, pragmáticos modernistas, y un movimiento -expresado tanto por graduados y estudiantes- que se plantea generar una antropología con perspectivas nacionales y latinoamericanas" (Garbulsky, 2004, p. 41). La orientación fundamental en la UNL tenía sus marcadas diferencias con lo que ocurría en la UBA, en donde existía una hegemonía de los enfoques de la denominada "escuela histórico-cultural".

Rosario consolidó una formación en la que los antropólogos y los arqueólogos trabajaban juntos incorporando todas las dimensiones de la vida humana (Achilli, 2011), insistiendo en la obligación de estructurar en una totalidad organizada y plantear los problemas de la realidad argentina (Petruzzi, 1965, p. 51, tomado de Garbulsky, 2004, p. 41). Esto se verá expresado en la participación de Petruzzi, docente titular de la asignatura "Antropología Cultural" desde donde introdujo la enseñanza del estructuralismo en Rosario -excluido de la formación oficial en la UBA-, en el Seminario de Técnicas de la Investigación compartido con arqueólogos y bioantropólogos. Además, en este clima de época se produjo una fuerte articulación entre el Instituto de Antropología y distintas dependencias de la Universidad, traducida en la realización de proyectos en conjunto con el Instituto de Planeamiento Regional y Urbano y la Facultad de Ciencias Económicas.

En las expediciones, los equipos recuperaron piezas arqueológicas y regresaron a la ciudad con una variada serie de documentos generados en torno a las tareas realizadas. En su rol como docente y director del Instituto de Antropología de la UNL, González coordinó las dos primeras expediciones arqueológicas a El Alamito, realizadas en marzo-abril de 1957 y febrero-marzo de 1958: "En verano. Porque era la época en que nosotros no trabajábamos en la facultad" (Entrevista realizada a María Teresa Carrara, 24 de octubre de 2017). En la "1 Expedición Arqueológica al Noroeste Argentino" se localizaron los sitios de la "Mesada del Arbolito", sobre la base de un croquis obtenido por calco de las fotografías aéreas realizadas por González algunos años antes. Identificaron los sitios con letras correlativas acompañadas del número cero (Núñez Regueiro, 1998). Tras la partida de González hacia Estados Unidos, las exploraciones realizadas el verano de 1958 estuvieron bajo la coordinación en campo de Petruzzi y pretendieron buscar respuestas para los interrogantes ya planteados previos a la primera salida.

\section{Excavaciones en el Museo}

La importancia del museo radica en que su creación se constituyó en un hecho central en el proceso de profesionalización de la disciplina antropológica en Rosario. Allí se desarrollaron instancias de aprendizaje de temas específicos, se comentaron diferentes textos y autores y se conformó un espacio donde los profesores y los estudiantes se conocían personalmente. Además, los docentes convocaban a los estudiantes interesados en participar o colaborar en investigaciones (Soprano, 2010). La creación del museo favoreció la realización de investigaciones de campo sistemáticas, en tanto existía un espacio donde analizar los objetos recuperados en las expediciones, sistematizar la información relevada y comenzar a diagramar las publicaciones que serían incluidas en el primer número de la Revista del Instituto de Antropología publicado en 1959.

Excavar el Museo implica ordenar y sistematizar distintos elementos dispersos, revalorizando una colección, restableciendo vinculaciones por sobre las discontinuidades históricas de la Antropología rosarina. La potencialidad analítica del "Fondo documental El Alamito" se ve expresada en los emergentes que surgen a partir del entrecruzamiento de sus distintos componentes. En este sentido, me vi frente a dos situaciones antagónicas: la presencia o la ausencia de una pieza arqueológica (Figura 1).

Para dar cuenta de los casos en los que los objetos arqueológicos pudieron ser ubicados, seleccioné el menhir (Figura 2). Elegí esta pieza porque siempre me llamó la atención el modo en que los miembros del Museo la mencionaban y los cuidados particulares que le brindaban, "El menhir que tenemos acá, ¿te acordás? Estaban entre medio...nuestro adorado menhir, está durmiendo, ahora tiene cajoncito, esta cuidadito" (Intervención de Nélida de Grandis en entrevista realizada a María Teresa Carrara, 24 de octubre de 2017).

Por otra parte, el relevamiento de los elementos del Fondo me permitió comprender que este singular accionar en relación con el menhir podía ser rastreado desde el momento mismo de su hallazgo. El interés que los investigadores depositaron en el menhir se puede leer en las crónicas periodísticas de la época, en las que ocupaba un lugar central, y era fotografiado y descripto 


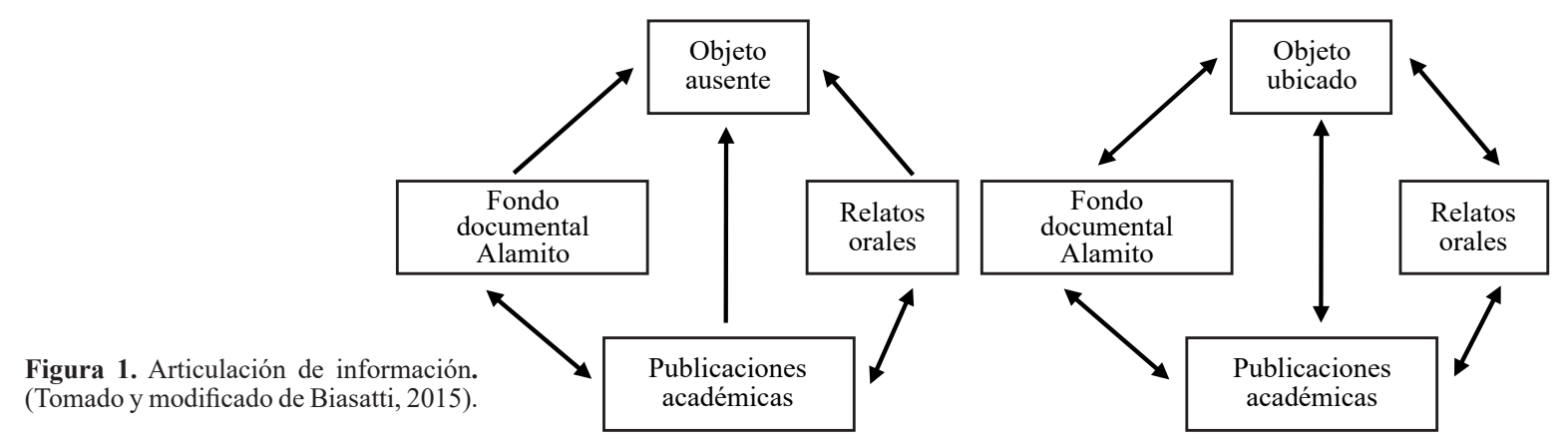

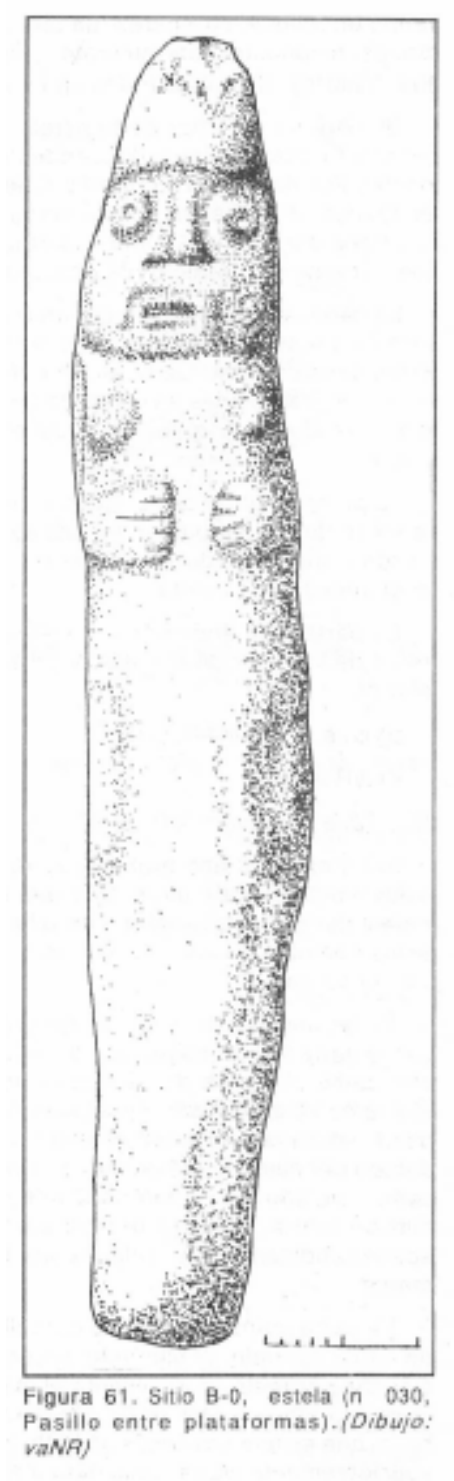

Figura 2. Dibujo del menhir realizado por Víctor Núñez Regueiro (1998, p. 83). con un detenimiento que no se repetía con ningún otro hallazgo.

Comencé entonces a buscar posibles explicaciones para este trato diferencial. Me pregunté qué era ese objeto, qué implicancias tenía o tiene que sea considerado un elemento de culto, si su carga ritual explica el trato. Retomando los postulados de la arqueología simétrica, "la acción no es algo generado por un agente identificable como tal (un sujeto, un ser humano) sino algo que se da dentro de una red de eventos y transformaciones cuya estructura se halla en constante cambio" (Loredo Narciandi, 2009, p. 119). Es decir, lo que tradicionalmente se ha considerado sujetos o agentes -en tanto centros de actividad- así como los objetos -las cosas, la naturaleza, los artefactos- forman parte de esa red en pie de igualdad: son producidos por su funcionamiento, no preexisten a ella. Empleando una terminología más próxima a la de Latour, "el mundo es como una red de acciones cuyos nodos, a veces cambiantes, son "actores" o "actantes", esto es, "humanos" o "no humanos", respectivamente" (Loredo Narciandi, 2009, p. 120). Desde esta perspectiva, el menhir debe ser analizado como un elemento en el que convergen redes de sentidos, sentidos que aparecen porque existe una serie de relaciones, algunas fijas, otras móviles: diversas clases de relaciones que hacen del menhir un menhir (Laguens, 2008).

La posibilidad de relacionar elementos dispersos a partir de la noción de fondo documental también se puede pensar en los casos en que los componentes dan cuenta de una pieza arqueológica que, por el momento, no se puede hallar. En este sentido, resulta ilustrativo el caso de la urna funeraria encontrada a la vera de la Ruta Nacional $\mathrm{N}^{\circ} 65$, mencionado en el anterior apartado. En el relevamiento de las fichas de Catamarca pude identificar la correspondiente a esta pieza, numerada como 59/14. Es decir, si bien cuento con una ficha y un cuaderno, dos elementos que describen la urna y detallan con precisión sus características y el contexto de su hallazgo, al no poder ubicar el ovejo, ¿deja de estar presente?, ¿la presencia/ausencia está dada por su corporalidad?, ¿no es un dato en sí mismo su ausencia?, ¿el entrecruzamiento de distintos elementos no instala acaso la pregunta por la presencia de ese objeto?

\section{Bibliografía}

Achilli, E. (2011). El quehacer antropológico en Rosario. Experiencias formativas generacionales y huellas de una antropología crítica. Revista de la Escuela de Antropología (UNR), XVII, 23-34. 
Achilli, E. (2014). De una antropología crítica en Rosario: Edgardo Garbulsky. En S. Sánchez (Comp.), Antropología crítica. Edgardo Garbuslky (pp. 11-17). Rosario: Laborde editor.

Achilli, E. (2015). Una práctica antropológica. De aprendizajes y reflexividad. Conferencia magistral en el marco de las V Jornadas de Antropología Social del Centro, "Antropología Social y Mundos Posibles en Transformación". https:/www.youtube.com/watch?v=NAE2yrOrq1c (Acceso: 12 de junio de 2016).

Bianchi, S. y Silvano, C. (2001). El oficio del cientista hoy... desde lo siniestro a lo ético político. Revista de la Escuela de Antropología (UNR), IV, 23-28.

Biasatti, S. (2015). Informe Final del Proyecto "Historia de las colecciones del Área Antropología y Paleontología del Museo Gallardo" (inédito).

Garbulsky, E. (2000). La historia de la antropología en Rosario. Reflexiones teórico-metodológicas. En S. Sánchez (Comp.), Antropología crítica. Edgardo Garbuslky (pp. 139-154). Rosario: Laborde.

Garbulsky, E. (2004). La producción del Conocimiento Antropológico-Social en la Facultad de Filosofía y Letras de la Universidad Nacional del Litoral, entre 1956-1966. Vínculos y relaciones nacionales. Cuadernos de Antropología Socia ( Instituto de Ciencias Antropológicas de la UBA) 20, 41-60.

Loredo Narciandi, J.C. (2009). ¿Sujetos o “actantes”? El constructivismo de Latour y la psicología constructivista. AIBR Revista de Antropología Iberoamericana, 4 (1), 113136.

Laguens, A. (2008). Objetos en objetos. Hacia un análisis relacional de lo estético en Arqueología. Iconica Antiquitas, $1-11$.

Nuñez Regueiro, V. (1998). Arqueología, historia y antropología de los sitios de Alamito. San Miguel de Tucumán: Ediciones INTERDEA.

Pegoraro, A. y Spoliansky, V. (2013). El Archivo del Museo Etnográfico Juan B. Ambrosetti: documentos para la historia institucional y disciplinar. Revista Electrónica de Fuentes y Archivos Archivos (Centro de Estudios Históricos "Prof. Carlos S. A. Segreti”, Córdoba), 4, 180-189.

Podgorny, I. (2011). Fronteras de papel: archivos, colecciones y la cuestión de los límites en las naciones americanas. Historia Crítica 44, 56-79.

Pupio, M. A. (2013). Archivos para una historia de la práctica de la arqueología. Revista Electrónica de Fuentes y Archivos (Centro de Estudios Históricos "Prof. Carlos S. A. Segreti", Córdoba), 4 (4), 24-33.

Soprano, G. (2010). La enseñanza de la arqueología en la Facultad de Ciencias Naturales y Museo de la Universidad Nacional de La Plata. Un análisis sobre el liderazgo académico de Alberto Rex González y Eduardo Mario Cigliano (19581977). Revista del Museo de Antropología (Universidad Nacional de Córdoba), 3, 171-186. 